\title{
Sex Differences in the Morphology of RR-Matched T-waves
}

\author{
Julia Ramírez ${ }^{1}$, Stefan van Duijvenboden ${ }^{1}$, Andrew Tinker ${ }^{1}$, Pier D Lambiase ${ }^{2}$, Patricia B Munroe ${ }^{1}$, \\ Michele Orini ${ }^{2,3}$ \\ ${ }^{1}$ William Harvey Research Institute, Queen Mary University of London, London, United Kingdom \\ ${ }^{2}$ Institute of Cardiovascular Science, University College London, London, United Kingdom \\ ${ }^{3}$ Barts Heart Centre, St Bartholomew's Hospital, London, United Kingdom
}

\begin{abstract}
Evidence of sex-related differences in cardiac risk is emerging, but whether these reflect sex-related differences in ventricular electrophysiology remains unclear. Our aim was to quantify T-wave morphological differences between men and women across different leads and RR interval values. We analysed 12-lead ECG recordings from 23,962 participants in the UK Biobank without known cardiovascular disease, and subsequently clustered them into bins of RR interval. In each cluster, we derived a lead and sexspecific mean warped T-wave (MWT). Then, we quantified differences between MWT in men and women in time and amplitude using linear, $d_{w}$ and $d_{a}$, and non-linear markers, $d_{w}^{N L}$ and $d_{a}^{N L}$. Leads V3 and aVR showed the lowest differences between men and women (median $d_{w}, d_{w}^{N L}, d_{a}$ and $d_{a}^{N L}$ of $1.12 \mathrm{~ms}, 0.69 \mathrm{~ms}, 3.29$ and 1.20 , respectively), while V1 showed the largest (5.69 ms, $4.50 \mathrm{~ms}, 208.94$ and 199.45 , respectively). Sex-related differences in MWT increased with the RR interval $\left(d_{w}, d_{w}^{N L}, d_{a}\right.$ and $d_{a}^{N L}$ ranging 1.44 - $5.89 \mathrm{~ms}, 1.23-3.97 \mathrm{~ms}, 8.58-28.38$ and 1.53 - 4.41, respectively). These values compare to those found for morphological T-wave variations due to large changes in heart rate (5.66 ms, $2.35 \mathrm{~ms}, 57.61$ and 9.51, respectively). These results indicate sex and lead should be considered when using $T$-wave morphologies for cardiovascular risk prediction.
\end{abstract}

\section{Introduction}

Sudden cardiac death (SCD) is a devastating event, occurring before any diagnosis of underlying heart disease in $50 \%$ of cases [1]. Higher rates have been reported in men than in women [1], indicating gender plays an important role in the initiation and development of malignant ventricular arrhythmias.

Several electrocardiogram (ECG) markers of ventricular repolarization are strongly associated with $\mathrm{SCD}$, including the corrected QT interval, the T-peak-to-T-end (Tpe) interval, and their dynamics $[2,3]$. All these ECG markers have been reported to be significantly different between men and women in the general population [4] and in cohorts with an underlying cardiovascular condition $[3,5]$. However, these predictors are based on specific features of the T-wave, so the impact that sex has on the overall T-wave morphology still remains unclear.

A novel methodology based on time-warping was recently proposed to quantify changes in T-wave morphology [6]. Using this methodology, the T-wave morphology restitution (TMR) index, quantifying the level of T-wave morphology variations with heart rate, demonstrated to be a stronger predictor of SCD in chronic heart failure patients $[7,8]$, and of ventricular arrhythmias in the general population [9] than traditional ECG indices of ventricular repolarization.

The aim of this study was to use time-warping methods [6] to detect and quantify sex-differences in the T-wave morphology in different leads in a large cohort from the general population.

\section{Materials and Methods}

\subsection{Materials}

UK Biobank (UKB, www.ukbiobank.ac.uk) is a prospective study of 488,377 individuals comprising relatively even numbers of men and women aged 40 to 69 years old at recruitment (2006 - 2008). A sub-cohort of 23,962 individuals participated in an imaging study (2014 - present, the collection is ongoing), which included 10seconds 12-lead ECG recordings.

The UKB study was approved by the North West MultiCentre Research Ethics Committee and all participants provided written informed consent.

\subsection{Methods}

\subsubsection{ECG Pre-processing and clustering}

Pre-processing of the ECG signals included low-pass filtering at $50 \mathrm{~Hz}$ to remove electric and muscle noise but still 
allow QRS detection [10]. Baseline wander was removed by further high-pass filtering the ECG signals at $0.5 \mathrm{~Hz}$.

We then signal-averaged the heartbeats to attenuate noise and artifacts and reveal small variations in the QRS and $\mathrm{T}$ waveforms. The onset, peak, and end timings of the waveforms were located using bespoke software $[10,11]$. The T-waves were selected from the T-wave onset and Twave end timing locations.

We then split the participants into men $(\mathrm{N}=11,007$, $45.9 \%)$ and women $(\mathrm{N}=12,955,54.1 \%)$, and subsequently clustered them into bins of RR interval (width of $50 \mathrm{~ms}$, range $0.625-1.325 \mathrm{~s}$ ).

\subsubsection{Mean Warped T-waves}

In each cluster, we derived an RR-matched mean warped T-wave (MWT). For this, we calculated an initial MWT that is an optimal representative average both in temporal and amplitude domains. The methodology is explained in detail in [6]. We, then, re-calculated the MWT by only considering those T-waves highly correlated (Spearman's correlation coefficient $>0.98$ ) with the initial MWT.

\subsubsection{Quantification of morphological changes}

Let $\boldsymbol{f}^{r}\left(\boldsymbol{t}^{m}\right)$ and $\boldsymbol{f}^{w}\left(\boldsymbol{t}^{w}\right)$ be two MWTs (i.e. men and women), where $\boldsymbol{t}^{m}$ and $\boldsymbol{t}^{w}$ are the temporal vectors of length $N_{r}$ and $N_{s}$, respectively, of each MWT. Let $\gamma\left(\boldsymbol{t}^{m}\right)$ be the warping function that relates $\boldsymbol{t}^{m}$ and $\boldsymbol{t}^{w}$, such that the composition $\boldsymbol{f}^{w}\left(\gamma\left(\boldsymbol{t}^{m}\right)\right)$ denotes the time domain warping of $\boldsymbol{f}^{w}\left(\boldsymbol{t}^{w}\right)$ using $\gamma\left(\boldsymbol{t}^{m}\right)$.

As demonstrated in [12], the optimal warping function can be obtained using the dynamic programming algorithm [13] as:

$$
\boldsymbol{\gamma}^{*}\left(\boldsymbol{t}^{m}\right)=\underset{\boldsymbol{\gamma}\left(\boldsymbol{t}^{m}\right)}{\arg \min }\left(\left\|\boldsymbol{q}_{f^{m}}\left(\boldsymbol{t}^{m}\right)-\boldsymbol{q}_{f w}\left(\boldsymbol{\gamma}\left(\boldsymbol{t}^{m}\right)\right) \sqrt{\dot{\boldsymbol{\gamma}}\left(\boldsymbol{t}^{m}\right)}\right\| \boldsymbol{j}^{1}\right)
$$

The metric $d_{w}$ quantifies the level of warping needed to optimally align any two MWT [6]:

$$
d_{w}=\frac{1}{N_{r}} \sum_{n=1}^{N_{r}}\left|\gamma^{*}\left(t^{m}(n)\right)-t^{m}(n)\right|
$$

The normalised amplitude difference between $\boldsymbol{f}^{m}\left(\boldsymbol{t}^{m}\right)$ and $\boldsymbol{f}^{w}\left(\boldsymbol{\gamma}^{*}\left(\boldsymbol{t}^{m}\right)\right)$ is quantified as:

$$
d_{a}=\frac{\frac{1}{N_{r}} \sum_{n=1}^{N_{r}} f^{w}\left(\gamma^{*}\left(t^{m}(n)\right)\right)-f^{m}\left(t^{m}(n)\right)}{\frac{1}{N_{r}} \sum_{n=1}^{N_{r}} f^{m}\left(t^{m}(n)\right)} \times 100
$$

By fitting $\gamma^{*}\left(t^{m}(n)\right)$ with a linear regression, $\gamma_{l}^{*}\left(t^{m}(n)\right)$, we have a quantification of the level of nonlinear warping:

$$
d_{w}^{\mathrm{NL}}=\frac{1}{N_{r}} \sum_{n=1}^{N_{r}}\left|\gamma^{*}\left(t^{m}(n)\right)-\gamma_{l}^{*}\left(t^{m}(n)\right)\right|
$$

By normalizing the warped MWTs, non-linear amplitude differences not due to linear scaling can be quantified as:

$$
d_{a}^{\mathrm{NL}}=\left\|\frac{f^{m}\left(t^{m}\right)}{\left\|f^{m}\left(t^{m}\right)\right\|}-\frac{f^{w}\left(\gamma^{*}\left(t^{m}\right)\right)}{\left\|f^{w}\left(\gamma^{*}\left(t^{m}\right)\right)\right\|}\right\| \times 100 .
$$

The morphological differences between men and women for each lead- and RR-specific MWT were quantified using $d_{w}, d_{w}^{N L}, d_{a}$ and $d_{a}^{N L}$ [6]. Therefore, there was a value of $d_{w}, d_{w}^{N L}, d_{a}$ and $d_{a}^{N L}$ for each lead and RR bin.

\subsubsection{Statistical Analyses}

To test if there were significant sex-differences across leads, we applied the Kruskal-Wallis test (a non-parametric one-way analysis of variance) to each morphological index $\left(d_{w}, d_{w}^{N L}, d_{a}\right.$ and $\left.d_{a}^{N L}\right)$. Then, we used box-plots to display relevant differences.

Similarly, to test if there were significant sex-differences across RR interval values, we applied the Kruskal-Wallis test to each morphological index. We also used box-plots to display relevant differences across values of RR. A P < 0.05 was considered statistically significant.

As described in the introduction, intra-individual variations in T-wave morphology as a response to heart rate changes are a strong cardiovascular risk predictor [7-9]. To investigate if the variations in the morphology due to sex differences were within a similar range than those due to changes in RR interval, we additionally quantified, for each lead and sex, the T-wave morphological differences between the two MWTs located at $0.625 \mathrm{~s}$ and $1.325 \mathrm{~s}$, respectively (extreme RR interval values).

\section{Results}

The average percent of T-waves excluded in the calculation of the MWT (section 2.2.2) across clusters was low $(2 \%)$.

\subsection{Differences across leads}

Kruskal-Wallis test was significant when testing across leads for the four morphological markers $\left(P=1 \times 10^{-15}\right.$ for $d_{w}, P=2 \times 10^{-17}$ for $d_{w}^{N L}, P=2 \times 10^{-23}$ for $d_{a}$, and $P=7 \times 10^{-5}$ for $d_{a}^{N L}$, Figure 1). 

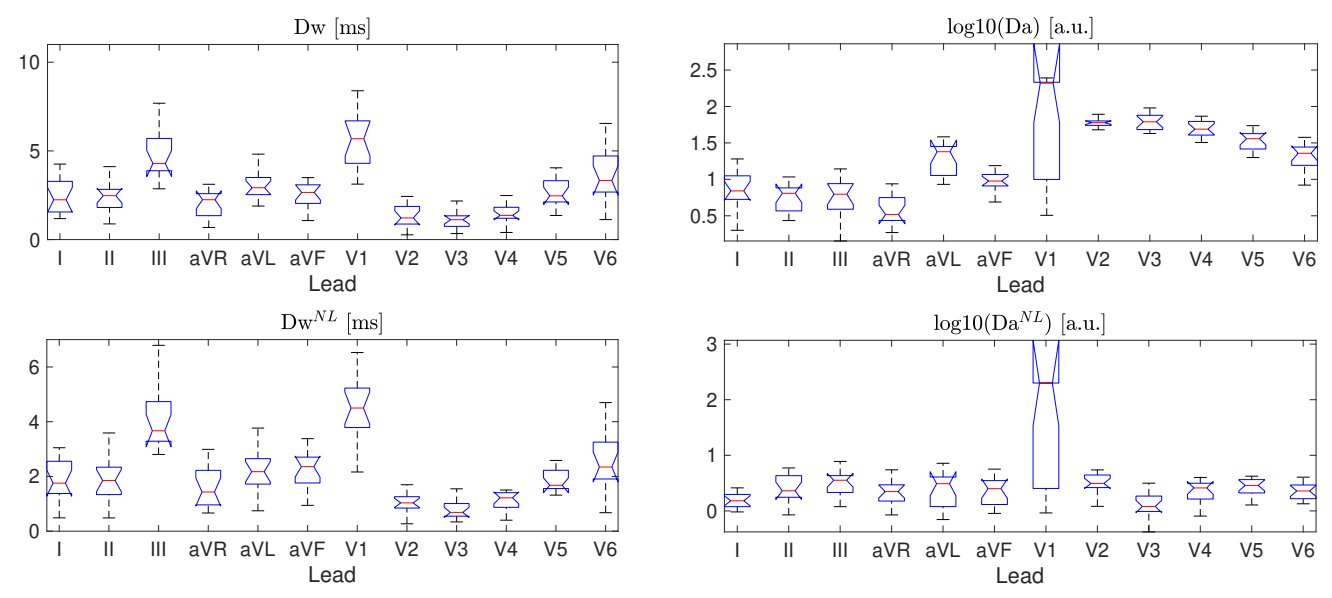

Figure 1. Sex-differences in T-wave morphology across leads. Boxplots of $d_{w}$ (top left panel), $d_{a}$ (top right panel), $d_{w}^{N L}$ (bottom left panel) and $d_{a}^{N L}$ (bottom right panel) for each lead. The logarithmic scale has been used for $d_{a}$ and $d_{a}^{N L}$ to facilitate comparison across leads.

Differences between men and women were lowest in $\mathrm{V} 3\left(d_{w}=1.12[0.60] \mathrm{ms}\right.$, median [interquartile range $\mathrm{IQR}])$, and largest in V1 $\left(d_{w}=5.69[2.39] \mathrm{ms}\right)$. Regarding morphological variations in the amplitude domain, aVR showed the lowest difference $\left(d_{a}=3.29\right.$ [2.92] a.u.), whereas $\mathrm{V} 1$ showed, again, the largest differences $\left(d_{a}=\right.$ 208.94 [204.48], Figure 1). Similar results were found for the non-linear markers $d_{w}^{N L}$ and $d_{a}^{N L}$ (Figure 1).

\subsection{Differences across $R R$ interval values}

Regarding sex-differences across RR interval values, Kruskal-Wallis test indicated statistically significant differences for $d_{w}\left(P=2 \times 10^{-2}\right)$, and for $d_{a}^{N L}\left(P=5 \times 10^{-2}\right)$, but not for $d_{w}^{N L}\left(P=8 \times 10^{-2}\right)$, nor for $d_{a}\left(P=1 \times 10^{-0}\right.$, Figure 2).

The index $d_{w}$ was lowest at RR of $0.875 \mathrm{~s}(1.44$ [1.23] $\mathrm{ms})$, and largest at RR of $1.325 \mathrm{~s}(5.89$ [3.40] ms), while $d_{a}^{N L}$ was lowest at RR of 0.625 (1.53 [1.57]) and largest at $\mathrm{RR}$ of $1.275 \mathrm{~s}$ (4.41 [3.05], Figure 2).

\subsection{Differences due to $\mathbf{R R}$ changes}

Table 1 shows the values of $d_{w}, d_{a}, d_{w}^{N L}$ and $d_{a}^{N L}$ when measuring TWMs variations at extreme RR values in men and in women. As shown, the values of $d_{w}$ and $d_{w}^{N L}$ are within a similar range than those shown in Figures 1 and 2. However, the changes in the amplitude domain with heart rate, as captured by $d_{a}$ and $d_{a}^{N L}$, are higher than the changes due to sex differences.

\begin{tabular}{lcc}
\hline & Men & Women \\
\hline$d_{w}$ & $5.66(1.79)$ & $5.34(1.43)$ \\
$d_{a}$ & $57.61(38.18)$ & $31.12(17.59)$ \\
$d_{w}^{N L}$ & $2.35(1.71)$ & $2.80(1.23)$ \\
$d_{a}^{N L}$ & $9.51(4.20)$ & $5.14(6.88)$ \\
\hline
\end{tabular}

Table 1. Median (interquartile range) values of the four morphological markers between two T-waves measured at extreme RR interval values in men and women.

\section{Discussion and Conclusions}

The main finding of this work is that sex impacts T-wave morphology, in addition to its duration. We observed that the impact of sex on the T-wave morphology was highly dependent on the lead where the T-wave was measured, whereas sex-differences were consistent over a wide range of RR intervals. The extremely large morphological differences observed in lead V1 might be explained by the fact that $\mathrm{T}$-waves in this lead are mainly biphasic, thus, having the largest morphological heterogeneity. Moreover, precordial electrode placement and anatomical differences in women might have played a role in our results.

When comparing morphological differences due to sex with those due to extreme RR changes, we observed differences in the temporal domain were comparable, indicating that sex can potentially influence the quantification of restitution of dispersion of repolarization, which is strongly associated with cardiovascular risk [7-9].

In addition, our results suggest the morphology markers, $d_{w}, d_{w}^{N L}, d_{a}$ and $d_{a}^{N L}$ could be used to predict the sex from the T-wave morphology, as previously done using artificial intelligence [14], or to estimate drug cardiotoxicity, following previous studies reporting abnormalities in the T-wave morphology [15]. 

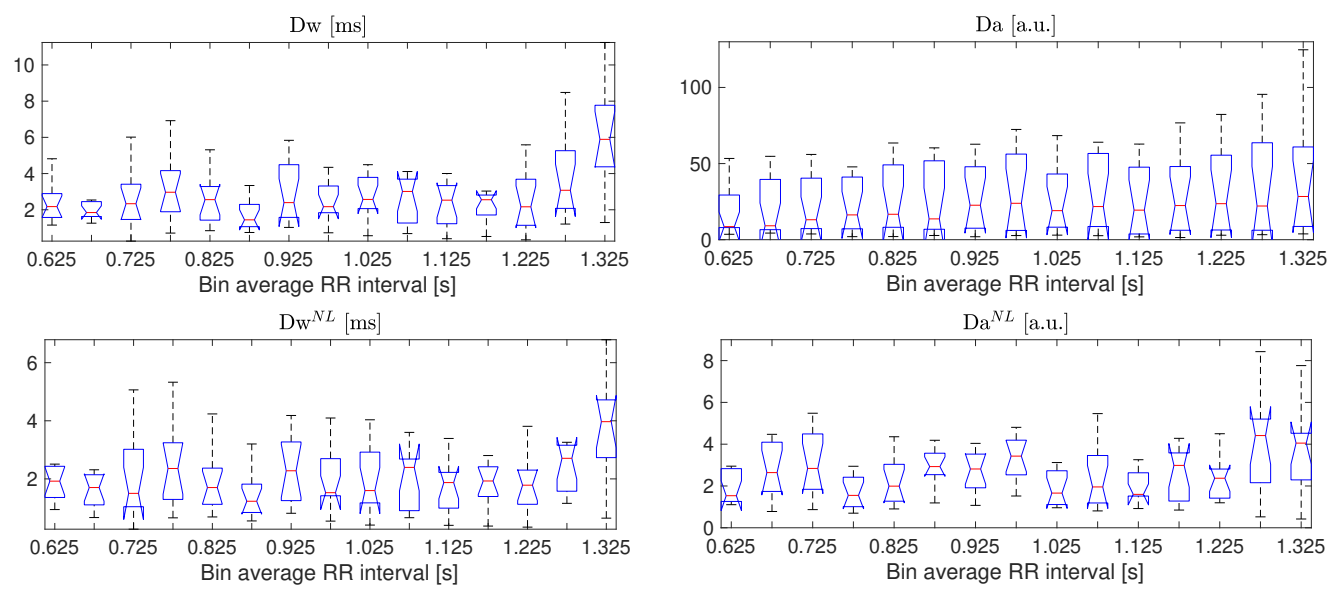

Figure 2. Sex-differences in T-wave morphology across RR interval values. Boxplots of $d_{w}$ (top left panel), $d_{a}$ (top right panel), $d_{w}^{N L}$ (bottom left panel) and $d_{a}^{N L}$ (bottom right panel) for different RR interval values

In conclusion, sex influences the T-wave morphology in an unselected population, and sex-differences in the Twave morphology are comparable to or higher than differences due to RR changes, and markedly heterogeneous across leads. Sex should be considered when using T-wave morphologies for cardiovascular risk prediction, but further studies are needed to optimise markers of T-wave morphological differences for risk prediction.

\section{Acknowledgements}

This research has been conducted using the UK Biobank Resource (application 8256) and is supported by the MRC grant MR/N025083/1. JR acknowledges support from the People Programme (Marie Curie Actions) of the European Union's Seventh Framework Programme (FP7/2007/2013) under REA grant agreement 608765. PDL is supported by UCL/UCLH Biomedicine NIHR. We also wish to acknowledge the NIHR Cardiovascular Biomedical Research Unit at Barts and QMUL, UK.

\section{References}

[1] M. Hayashi et. al., "The spectrum of epidemiology underlying sudden cardiac death.," Circ Res, vol. 116, pp. 18871906, 2015.

[2] J. Ramírez et. al., "Prediction of sudden cardiac death in chronic heart failure patients by analysis of restitution dispersion.," in Computing in Cardiology, pp. 1-4, 2013.

[3] J. Ramírez et. al., "QT/RR and T-peak-to-end/RR curvatures and slopes in chronic heart failure: Relation to sudden cardiac death.," J Electrocardiol, vol. 47, no. 6, pp. 842848, 2014.

[4] P. Smetana et. al., "Sex differences in repolarization homogeneity and its circadian pattern," Am J Physiol Heart Circ Physiol, vol. 282, no. 5, pp. H1889-H1897, 2002.

[5] H. Mieszczanska et. al., "Gender-related differences in electrocardiographic parameters and their association with cardiac events after myocardial infarction," Am J Cardiol, vol. 101, pp. P20-P24, 2008.
[6] J. Ramírez et. al., "Variability of ventricular repolarization dispersion quantified by time-warping the morphology of the T-waves.," IEEE Trans Biomed Eng, vol. 64, no. 7, pp. 1619-1630, 2017.

[7] J. Ramírez et. al., "T-wave morphology restitution predicts sudden cardiac death in patients with chronic heart failure," J Am Heart Assoc, vol. 6, no. 5, p. e005310, 2017.

[8] J. Ramírez et. al., "Sudden cardiac death and pump failure death prediction in chronic heart failure by combining ECG and clinical markers in an integrated risk model," PLoS ONE, vol. 12, no. 10, p. e0186152, 2017.

[9] J. Ramírez et. al., "Cardiovascular predictive value and genetic basis of ventricular repolarization dynamics," Circ Arrhythmia Electrophysiol, vol. 12, p. e007549, 2019.

[10] Orini M. et. al., "Long-term intra-individual reproducibility of heart rate dynamics during exercise and recovery in the UK Biobank cohort," Plos One, vol. 12, p. e0183732, 2017.

[11] Orini M. et. al., "A time-varying nonparametric methodology for assessing changes in QT variability unrelated to heart rate variability," IEEE Trans Biomed Eng, vol. 65, pp. 1443-1451, 2018.

[12] J. Ramírez et. al., "An index for T-wave pointwise amplitude variability quantification.," Computing in Cardiology Conference (CinC), pp. 49-52, 2016.

[13] D. P. Bertsekas, Dynamic programming and optimal control, ch. 1. Belmont, MA, 1995.

[14] Z. I. Attia et. al., "Age and sex estimation using artificial intelligence from standard 12-lead ECGs," Circulation Arr Electrophysiol, vol. 12, p. e007284, 2019.

[15] J. P. Couderc et. al., "T-wave morphology abnormalities in benign, potent and arrhythmogenic Ikr inhibition," Heart Rhythm, vol. 8, no. 7, pp. 1036-1043, 2011.

Address for correspondence:

Julia Ramírez, Charterhouse Square, London EC1M 6BQ, United Kingdom j.ramirez@qmul.ac.uk 